\title{
An Active-Only Temperature-Insensitive Current-Mode Biquad Filter Based on Differentiator Structures Employing CCCCTAs
}

\author{
Supawat Lawanwisut ${ }^{1}$, Montree Siripruchyanun ${ }^{1 *}$, Winai Jaikla ${ }^{2}$ \\ ${ }^{1}$ Department of Teacher Training in Electrical Engineering, Faculty of Technical Education, \\ King Mongkut's University of Technology North Bangkok, Bangkok, Thailand \\ ${ }^{2}$ Department of Engineering Education, Faculty of Industrial Education, King Mongkut's \\ Institute of Technology Ladkrabang, Bangkok, Thailand \\ Email: ${ }^{*}$ mts@kmutnb.ac.th
}

Received February 14, 2013; revised March 15, 2013; accepted March 25, 2013

Copyright (C) 2013 Supawat Lawanwisut et al. This is an open access article distributed under the Creative Commons Attribution License, which permits unrestricted use, distribution, and reproduction in any medium, provided the original work is properly cited.

\begin{abstract}
This article presents an active-only current-mode universal biquad filter performing three standard functions: low-pass, high-pass and band-pass function, which can be readily modified to achieve the rest functions (band-stop and all-pass). The circuit principle is based on active-only circuit designed by using differentiators which are constructed from current controlled current conveyor transconductance amplifier (CCCCTA) cooperating with an internally frequency compensated operational amplifier (OA). The features of the circuit are that: the pole frequency and quality factor can be independently tuned via the input bias currents and it is ideally temperature-insensitive, its circuit description is very simple, consisting of 3 CCCCTAs and 2 operational amplifiers, and the proposed circuit is very appropriate for further developing into integrated circuit architecture. The PSpice simulation results are shown. The given results agree well with the theoretical anticipation.
\end{abstract}

Keywords: Active-Only; CCCCTA; Current-Mode; Biquad Filter

\section{Introduction}

An analog filter is an important building block, widely used for continuous-time signal processing. It can be found in many fields: including, communications, measurement, instrumentation, and control systems [1,2]. One of the most popular analog filters is a universal biquad filter, since it can simultaneously provide several functions in the same circuit topology. Recently, a universal filter working in current-mode has been more popular than the voltage-mode type. Since last two decades, there has been much effort to reduce the supply voltage of analog systems. This is due to the demand for portable and battery-powered equipment. Since a low-voltage operating circuit becomes necessary, the current-mode technique is ideally suited for this purpose. Actually, a circuit using the current-mode technique has many other advantages, such as, larger dynamic range, higher bandwidth, greater linearity, simpler circuitry and lower power

${ }^{*}$ Corresponding author. consumption $[3,4]$.

The synthesis and design of analog signal processing circuits using only active elements without passive elements are important in fully integrated circuit (IC) technology. This technique makes circuit become smaller chip area, lower power consumption, wider frequency range of operation and programmability [5-8], where the applications can be easily seen in many literatures, for example filter [7], oscillator [9], inductance simulator [10] and etc.

From the past, creation of differentiator circuit must use an inductor worked together with an active element which affected on circuit as large sized. So, it was not popular to create circuit with differentiator. But nowadays, we can design differentiator-based circuit without any inductor, and then causing a reduction in circuit sized smaller than creation circuit in the past. Biquadratic transfer function is widely used in order to synthesize the filters. Many kinds of filters can be realized based on only integrators as building blocks [11-14]. However, 
integrator circuit performs as a low-pass filter. At high frequency, stage-gain of integrator is decayed due to components bandwidth and integrator characteristic. In order to realize filter, magnitude response of filter in high-frequency would be unstable according to the mentioned characteristics. Differentiator circuit can perform as a high-pass filter that compensated with component bandwidth for stabilization of magnitude response in high-frequency [15].

A reported 5-terminals active element, namely current convey transconductance amplifier (CCTA) [16] has been proposed in 2005, it seems to be a versatile component in the realization of a class of analog signal processing circuits, especially analog frequency filters. It is a really current-mode element whose input and output signals are currents. In addition, the output current gain can be adjusted via input bias current. However, the parasitic resistance at current input port of the CCTA cannot be controlled. Recently, Siripruchyanun and Jaikla have proposed the modified-version CCTA, whose parasitic resistance at current input port can be controlled by an input bias current. It is newly named current controlled current conveyor transconductance amplifier (CCCCTA) [17]. It seems to be a useful building block, since many circuits and systems can be implemented by employing only single CCCCTA. Presently, the CCCCTA has been extensively used, such as filters $[18,19]$, oscillators $[20$, 21], inductance simulators [22], and etc.

From our recent survey, it is found that several implementations of current-mode universal biquad filters using active-only principle have been reported. Unfortunately, these reported circuits suffer from one or more of following weaknesses:

- Excessive use of the active elements [23,24].

- Lack of electronic adjustability [23,24].

- The pole frequency and quality factor cannot be independently tuned $[18,25]$.

The aim of this paper is to propose a new currentmode universal biquad filter. The features of proposed circuit are that: it employs 3 CCCCTAs and 2 internally frequency compensated operational amplifiers; the proposed universal biquad filter can provide 3 standard functions including low-pass, high-pass and band-pass functions in the same time without changing circuit topology, where the rest functions (band-stop and all-pass) can be readily obtained by small modification; the circuit description is very simple, which results in a small-size of the monolithic chip. In addition, it is electronically tunable and convenient to use. The quality factor and pole frequency can be electronically and independently adjusted. The PSpice simulation results are also shown, which are in correspondence with the theoretical analysis.

\section{Theory and Principle}

\subsection{Implementation Topology of the Proposed Filter}

The filter is designed by cascading current adder and the current-mode lossless differentiators fed-back by current amplifier whose gain of $k$ as systematically shown in Figure 1. From block diagram in Figure 1, its transfer function can be found to be

$$
\begin{gathered}
\frac{I_{L P}}{I_{\text {in }}}=\frac{\frac{1}{a b}}{s^{2}+\frac{s k}{b}+\frac{1}{a b}}, \\
\frac{I_{B P}}{I_{\text {in }}}=\frac{\frac{s}{b}}{s^{2}+\frac{s k}{b}+\frac{1}{a b}},
\end{gathered}
$$

and

$$
\frac{I_{H P}}{I_{\text {in }}}=\frac{s^{2}}{s^{2}+\frac{s k}{b}+\frac{1}{a b}} .
$$

From Equations (1)-(3), the pole frequency $\left(\omega_{0}\right)$ and quality factor $\left(Q_{0}\right)$ can be respectively expressed as

$$
\omega_{0}=\sqrt{\frac{1}{a b}},
$$

and

$$
Q=\frac{1}{k} \sqrt{\frac{b}{a}} .
$$

It is found that the pole frequency can be adjusted by either $a$ or $b$, by keeping their ratio to be constant where the quality factor can be tuned through $k$ without effecting the pole frequency.

\subsection{Basic Concept of CCCCTA}

The principle of the CCCCTA was firstly published in 2008 by Siripruchyanun and Jaikla [17]. The schematic symbol and the ideal behavioral model of the CCCCTA are shown in Figures 2(a) and (b), respectively. The characteristics of the ideal CCCCTA are represented by

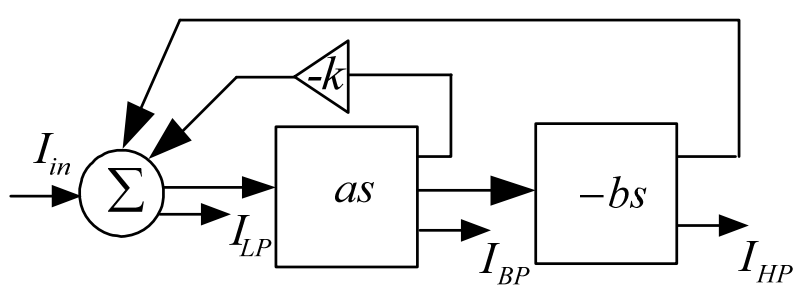

Figure 1. Block diagram for proposed filter implementation. 
the following equation:

$$
\left[\begin{array}{c}
I_{y} \\
V_{x} \\
I_{z, z_{c}} \\
I_{o}
\end{array}\right]=\left[\begin{array}{cccc}
0 & 0 & 0 & 0 \\
R_{x} & 1 & 0 & 0 \\
1 & 0 & 0 & 0 \\
0 & 0 & \pm g_{m} & 0
\end{array}\right]\left[\begin{array}{c}
I_{x} \\
V_{y} \\
V_{z} \\
V_{o}
\end{array}\right]
$$

If the CCCCTA is realized using a BJT technology, $R_{x}$ and $g_{m}$ can be respectively written as

$$
R_{x}=\frac{V_{T}}{2 I_{B 1}},
$$

and

$$
g_{m}=\frac{I_{B 2}}{2 V_{T}},
$$

where $g_{m}$ is transconductance of the CCCCTA. $V_{T}$ is the thermal voltage equal to $26 \mathrm{mV}$ at a room temperature. $I_{B 1}$ and $I_{B 2}$ are the bias currents used to control the parasitic resistance and transconductance of the CCCCTA. In general, CCCCTA can contain an arbitrary number of o terminals, providing currents $I_{O}$ of both directions, respectively. A possible BJT implementation of the CCCCTA is shown in Figure 3.

\subsection{Internally Frequency Compensated Operational Amplifier}

The open-loop gain of a practical internally frequency

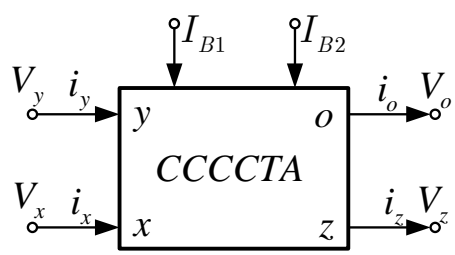

(a)

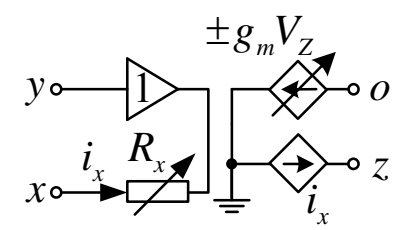

(b)

Figure 2. CCCCTA (a) Symbol (b) Equivalent circuit.

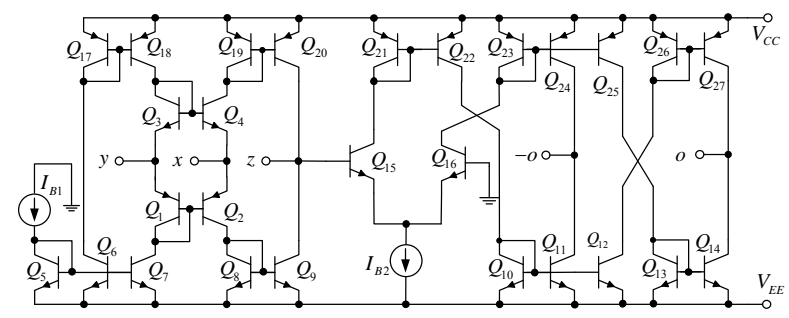

Figure 3. Schematic of the BJT CCCCTA. compensated operational amplifier $(O A)$ is represented by the following transfer function

$$
A(s)=\frac{A_{0} \omega_{p 1}}{s+\omega_{p 1}}=\frac{B}{s+\omega_{p 1}},
$$

where $A_{o}$ is open-loop DC gain, $\omega_{p 1}$ is the first pole frequency and $B\left(=A_{o} \omega_{p 1}\right)$ is the gain-bandwidth product of the operational amplifier. For the frequencies $\omega \gg \omega_{p 1}$, Equation (9) is approximately given by [5,7]

$$
A(s) \cong \frac{B}{s} \text {. }
$$

\section{Proposed Current-Mode Biquad Filter}

As mentioned in last section, the proposed filter is based on current amplifier and the current-mode lossless differentiators. In this section, these circuits will be described. The current amplifier based on the CCCCTA is shown in Figure 4. The output current of this circuit can be written to be

$$
I_{\text {OutA }}=k I_{\text {inA }},
$$

where $k=g_{m} R_{x} / 2$. Figure 5 shows the lossless differentiator using the CCCCTA. Considering the circuit in Figure 5 and using the CCCCTA properties, we will receive

$$
\frac{I_{\text {OutB }}}{I_{\text {inB }}}=s a,
$$

where $a=\frac{R_{x} g_{m}}{B}$.

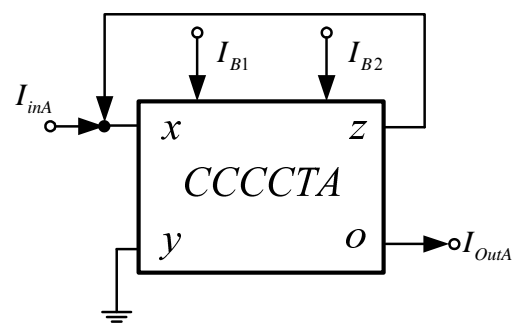

Figure 4. Current amplifier based on CCCCTA.

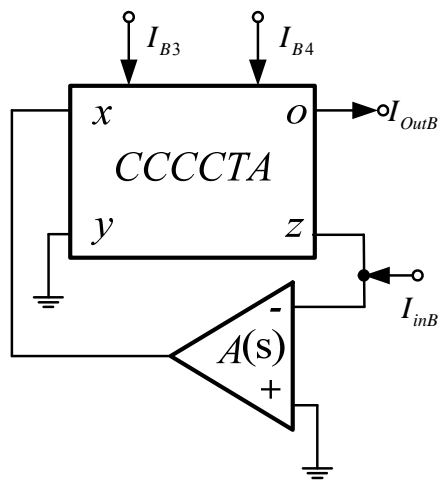

Figure 5. Lossless differentiator using CCCCTA and OA. 
The completed current-mode biquad filter is shown in Figure 6, based on filter topology in Figure 1. From Equation (12), if $B_{1}=B_{2}=B$ for easy consideration, the output current transfer functions of the circuit at each output terminal in Figure 6 can be obtained as

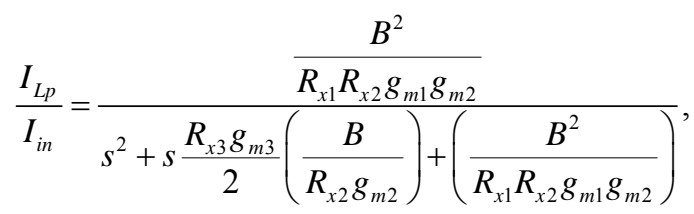

$$
\begin{aligned}
& \frac{I_{B p}}{I_{\text {in }}}=\frac{\frac{s B}{R_{x 2} g_{m 2}}}{s^{2}+s \frac{R_{x 3} g_{m 3}}{2}\left(\frac{B}{R_{x 2} g_{m 2}}\right)+\left(\frac{B^{2}}{R_{x 1} R_{x 2} g_{m 1} g_{m 2}}\right)}, \\
& \frac{I_{H p}}{I_{\text {in }}}=\frac{s^{2}}{s^{2}+s \frac{R_{x 3} g_{m 3}}{2}\left(\frac{B}{R_{x 2} g_{m 2}}\right)+\left(\frac{B^{2}}{R_{x 1} R_{x 2} g_{m 1} g_{m 2}}\right)} \text {, }
\end{aligned}
$$

Moreover, the band-stop and the all-pass functions can be further obtained, combining the currents $I_{B S}=$ $I_{H P}+I_{L P}$ and $I_{A P}=I_{B S}-I_{B P}$, respectively, where

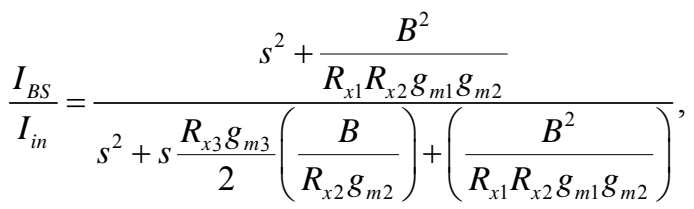

$$
\begin{aligned}
& \frac{I_{A P}}{I_{i n}}=\frac{s^{2}-\frac{s B}{R_{x 2} g_{m 2}}+\frac{B^{2}}{R_{x 1} R_{x 2} g_{m 1} g_{m 2}}}{s^{2}+s \frac{R_{x 3} g_{m 3}}{2}\left(\frac{B}{R_{x 2} g_{m 2}}\right)+\left(\frac{B^{2}}{R_{x 1} R_{x 2} g_{m 1} g_{m 2}}\right)} \text {. }
\end{aligned}
$$

The pole frequency $\left(\omega_{0}\right)$ and quality factor $\left(Q_{0}\right)$ can be respectively expressed to be

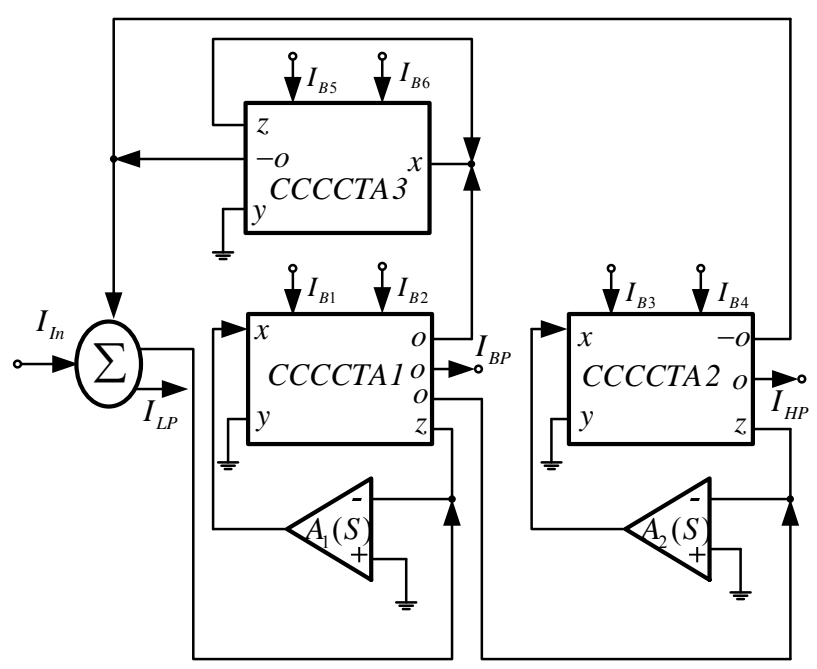

Figure 6. Completely proposed current-mode biquad filter.

$$
\omega_{0}=B \sqrt{\frac{1}{R_{x 1} R_{x 2} g_{m 1} g_{m 2}}},
$$

and

$$
Q=\frac{1}{k} \sqrt{\frac{R_{x 2} g_{m 2}}{R_{x 1} g_{m 1}}} .
$$

Substituting $R_{x 1}=V_{T} / 2 I_{B 1}, R_{x 2}=V_{T} / 2 I_{B 3}, R_{x 2}=$ $V_{T} / 2 I_{B 5}, g_{m 1}=I_{B 2} / 2 V_{T}, g_{m 2}=I_{B 4} / 2 V_{T}$ and $g_{m 3}=$ $I_{B 6} / 2 V_{T}$, the pole frequency and quality factor can be rewritten to be

$$
\omega_{0}=4 B \sqrt{\frac{I_{B 1} I_{B 3}}{I_{B 2} I_{B 4}}},
$$

and

$$
Q=\frac{8 I_{B 5}}{I_{B 6}} \sqrt{\frac{I_{B 1} I_{B 4}}{I_{B 2} I_{B 3}}} .
$$

It is obviously found that, from Equations (20) and (21), the pole frequency can be adjusted independently from the quality factor by varying either $I_{B 1}$ and $I_{B 3}$ or $I_{B 2}$ and $I_{B 4}$ (keeping their ratio to be constant), where the quality factor can be adjusted by $I_{B 5}$ or $I_{B 6}$ without affecting the pole frequency. In addition, if the gain-bandwidth product $(B)$ is independent of temperature variation, it is seen that the pole frequency and quality factor are ideally temperature insensitive.

\section{Sensitivity Analysis}

The sensitivities of the proposed circuit can be found as

$$
S_{I_{B 1}, I_{B 3}}^{\omega_{0}}=\frac{1}{2}, S_{I_{B 2}, I_{B 4}}^{\omega_{0}}=-\frac{1}{2}, S_{B}^{\omega_{0}}=1,
$$

and

$$
S_{B, I_{B S}}^{Q_{0}}=1, S_{I_{B 6}}^{Q_{0}}=-1, S_{I_{B 1}, I_{B 4}}^{Q_{0}}=\frac{1}{2}, S_{I_{B 2}, I_{B 3}}^{Q_{0}}=-\frac{1}{2}
$$

Therefore, all active and passive sensitivities are equal or less than unity in magnitude.

\section{Simulation Results}

The working performances of the proposed biquad filter were verified in PSpice simulation using the BJT implementation of the CCCCTA as shown in Figure 3. The PNP and NPN transistors employed in the proposed circuit were simulated by using the parameters of the PR200N and NR200N bipolar transistors of ALA400 transistor array from AT\&T [26]. The OA was simulated by using the parameter of TL082 with the gain-bandwidth product of $B=4 \mathrm{MHz}$ is used. The circuit was biased with $\pm 5 \mathrm{~V}$ power supplies voltage, $I_{B 1}=I_{B 3}=30 \mu \mathrm{A}, I_{B 2}=I_{B 4}=400 \mu \mathrm{A}, I_{B 5}=50 \mu \mathrm{A}$ and $I_{B 6}=100 \mu \mathrm{A}$, using ideal current summing circuit. The 
results shown in Figure 7 are the gain responses of the proposed biquad filter. It is clearly seen that it can provide low-pass, high-pass, band-pass functions without modifying any circuit topology. Figure 8 displays gain response for band-stop function as depicted in Equation (16). Figure 9 displays gain and phase responses for all-pass function analyzed in Equation (17).

Figure 10 displays gain responses of band-pass function, where $I_{B 1}$ and $I_{B 3}$ are equally set to keep its ratio to be constant and changed for several values. Figure 11 shows gain responses of band-pass function, where $I_{B 2}$ and $I_{B 4}$ are equally set to keep the ratio to be constant and changed for several values. Figures $\mathbf{1 2}$ and $\mathbf{1 3}$ are gain responses of band-pass function for different $I_{B 5}$ and $I_{B 6}$ values, respectively. It is shown that the bandwidth of the responses can be electronically adjusted by the input bias current $I_{B 5}$ and $I_{B 6}$ without affecting the pole frequency. Figure $\mathbf{1 4}$ shows gain re-

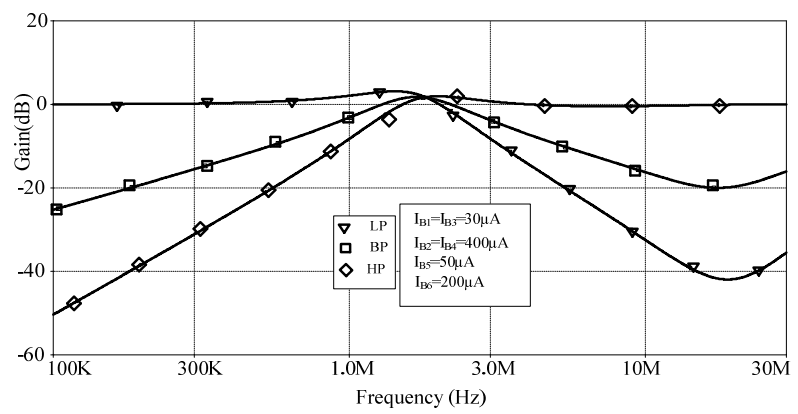

Figure 7. Gain responses of the proposed circuit.

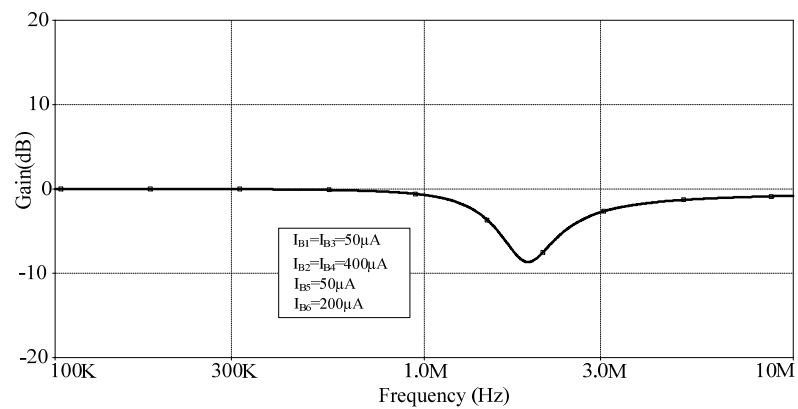

Figure 8. Gain response of the $I_{B S}$.

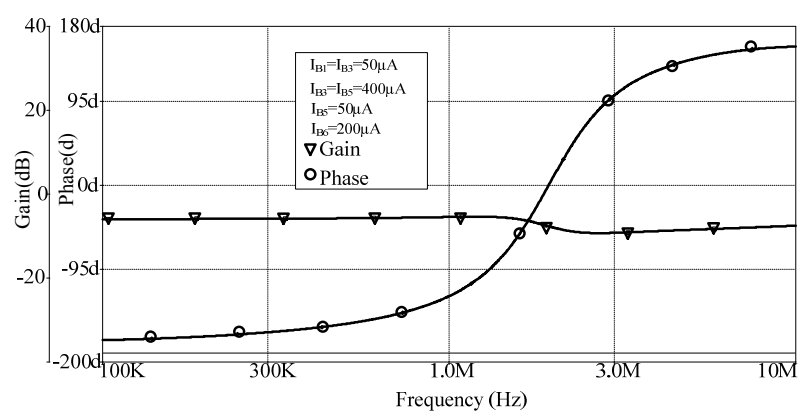

Figure 9. Gain and phase responses of the $I_{A P}$.

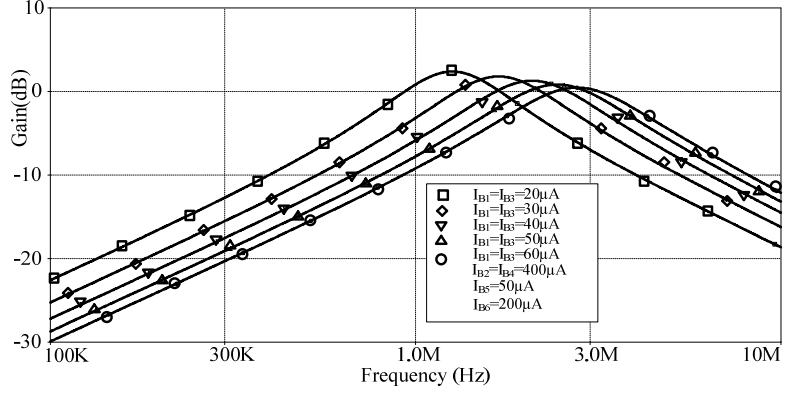

Figure 10. Band-pass responses for different values of $I_{B 1}$ and $I_{B 3}$ with keeping its ratio to be constant as $I_{B 1}=I_{B 3}=I_{B}$.

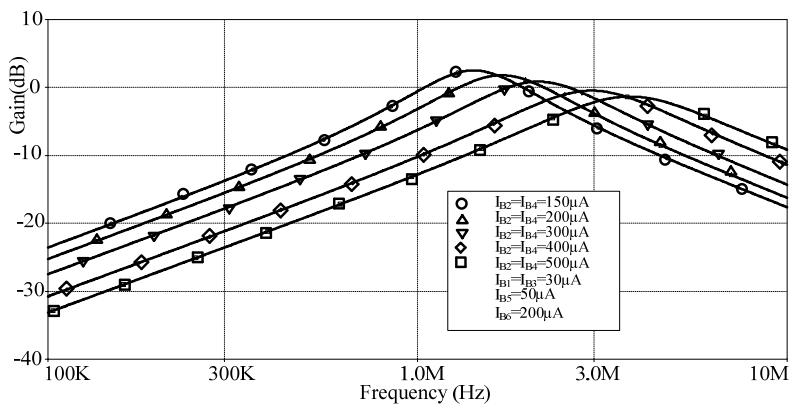

Figure 11. Band-pass responses for different values of $I_{B 2}$ and $I_{B 4}$ with keeping its ratio to be constant as $I_{B 2}=I_{B 4}=I_{B}$.

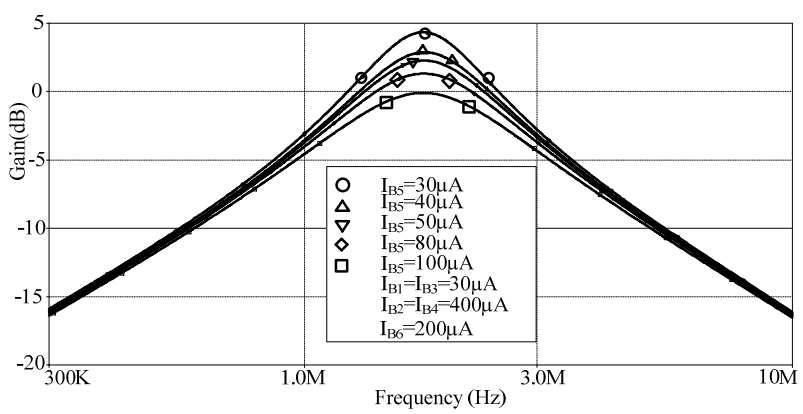

Figure 12. Band-pass responses for different values of $I_{B 5}$.

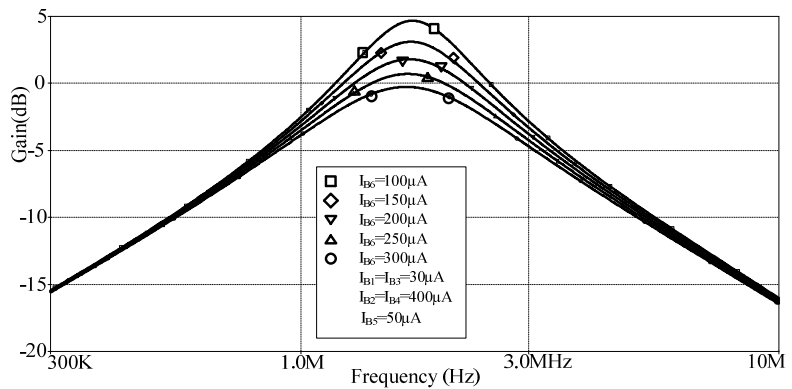

Figure 13. Band-pass responses for different values of $I_{B 6}$.

sponses of the band-pass function relative to different temperatures of $27^{\circ} \mathrm{C}, 50^{\circ} \mathrm{C}$ and $100^{\circ} \mathrm{C}$. From this result, it can be measured that the average deviation of parameters $\omega_{0}$ and $Q_{0}$ due to these temperature variations is 


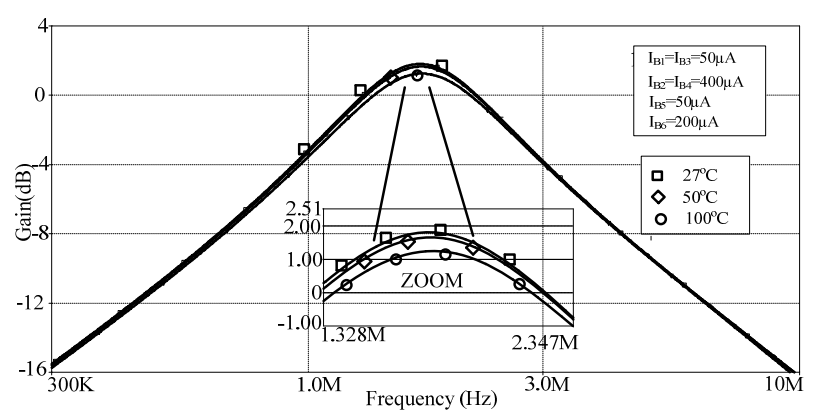

Figure 14. Band-pass responses for different temperature values.

merely $0.005196829 \% /{ }^{\circ} \mathrm{C}$. Consequently, it is concluded that the pole frequency and quality factor are slightly dependent on the temperature variations.

\section{Conclusion}

The current-mode biquad filter based on the differentiator configuration consisting of 3 CCCCTAs and 2 internally frequency compensated operational amplifiers has been presented. The advantages of the proposed circuit are that: it can simultaneously perform 3 standard functions, low-pass high-pass and band-pass functions from the same circuit configuration, where the rest functions (band-stop and all-pass) can be easily provided by small modification without component matching constraint and changing circuit topology; the pole frequency can be electronically tuned without affecting the quality factor with keeping their ratios constant. In addition, the pole frequency and quality factor are slightly temperature dependent. The PSpice simulation results were depicted, and agreed well with the theoretical anticipation. With mentioned features, it is very suitable to realize the proposed circuit in monolithic chip to use in battery-powered, portable electronic equipments such as wireless communication system devices.

\section{REFERENCES}

[1] C. A. S. Sedra and K. C. Smith, "Microelectronic Circuits," 5th Edition, Holt, Rinehart and Winston, Orlando, 2003.

[2] M. A. Ibrahim, S. Minaei and H. A. Kuntman, "A 22.5 $\mathrm{MHz}$ Current-Mode KHN-Biquad Using Differential Voltage Current Conveyor and Grounded Passive Elements," International Journal of Electronics and Communications, Vol. 59, No. 5, 2005, pp. 311-318. doi:10.1016/j.aeue.2004.11.027

[3] C. Toumazou, F. J. Lidgey and D. G. Haigh, "Analogue IC Design: The Current-Mode Approach,” Peter Peregrinus, Stevenage, 1990.

[4] C. Sakul, W. Jaikla and K. Dejhan, "New Resistor-Less Current-Mode Quadrature Oscillators Using 2 CCCDTAs and Grounded Capacitors," International Journal of Ra- dioengineering, Vol. 20, No. 4, 2011, pp. 890-897.

[5] M. T. Abuelma'atti and H. A. Alzaher, "Universal Three inputs and One output Current-mode Filter without External Passive Elements," Electronics Letters, Vol. 33, No. 4, 1997, pp. 281-283. doi:10.1049/el:19970233

[6] A. K Singh and R. Senani, "Low-Component-Count Active Only Immittances and Their Application in Realizing Simple Multifunction Biquads," Electronics Letters, Vol. 34, No. 8, 1998, pp. 718-719. doi:10.1049/el:19980547

[7] T. Tsukutani, M. Higashimura, Y. Sumi and Y. Fukul, "Electronically Tunable Current-Mode Active-Only Biquadratic Filter," International Journal of Electronics, Vol. 87, 2000, pp. 307-314. doi:10.1080/002072100132219

[8] M. T. Abbuelma'atti and M. A. Al-Qahtani, "Low Component Second-Generation Current Conveyor-Based Multiphase Sinusoidal Oscillator," International Journal of Electronics, Vol. 84, No. 1, 1998, pp. 45-52. doi:10.1080/002072198134986

[9] M. T. Abuelma'atti and H. A. Alzaher, "Active-Only Sinusoidal Oscillator," Elsevier Advanced Technology, Microelectronics Journal, Vol. 29, 1998, pp. 461-464. doi:10.1016/S0026-2692(97)00104-3

[10] S. Minaei, O. Cicekoglu, H. Kuntman and S. Turkoz, "Electronically Tunable, Active-Only Floating Inductance Simulation," International Journal of Electronics, Vol. 89, No. 12, 2002, pp. 905-912. doi:10.1080/0020721031000120470

[11] C. Chang and S. Pai, "Universal Current-Mode OTA-C Biquad with the Minimum Components," Proceedings of IEEE Transactions on Circuits and Systems, Vol. 47, No. 8, 2000, pp. 1235-1238. doi:10.1109/81.873877

[12] C. Chang, B. Al-Hashimi and J. N. Ross, "Unified Active Filter Biquad Structure," IEE Proceedings of Circuits, Devices and Systems, Vol. 151, No. 4, 2004, pp. 273-277. doi:10.1049/ip-cds:20040132

[13] M. T. Abuelma'atti and A. Bentrcia, "New Universal Current-Mode Multiple-Input Multiple-Output OTA-C Filter," The 2004 IEEE Asia-Pacific Conference on Circuits and Systems, Tainan, 6-9 December 2004, pp. 1037 1039.

[14] A. Fabre, O. Saaid, F. Wiest and C. Boucheron, "Current Controlled Bandpass Filter Based on Translinear Converyors," Electronics Letters, Vol. 31, No. 20, 1995, pp. 1727-1728. doi:10.1049/el:19951225

[15] M. Somdunyakanok, "Three Inputs Single Output Current mode Universal Filter," Proceeding of the 2009 12th IEEE International Analog VLSI Workshop, Chiang Mai, 18-20 November 2009, pp.113-116.

[16] R. Prokop and V. Musil, "New Modern Circuit Block CCTA and Some Its Applications," Proceedings the Fourteenth International Scientific and Applied Science Conference-Electronics, TU Sofia, Sofia, 2005, pp. 93-98.

[17] M. Siripruchyanun and W. Jaikla, "Current Controlled Current Conveyor Transconductance Amplifier (CCCCTA): A Building Block for Analog Signal Processing," International Journal of Electrical Engineering, Vol. 90, No. 6, 2008, pp. 443-453. 
[18] S. Lawanwisut and M. Siripruchyanun, "An Active-Only Current-Mode Electronically Controllable Multifunction Biquadratic Filter Using CCCCTAs," Proceedings of the 33rd Electrical Engineering Conference, 2010, pp. 10971100.

[19] W. Jaikla, A. Noppakarn and S. Lawanwisut, "New Gain Controllable Resistor-Less Current-Mode First Order Allpass Filter and Its Application," International Journal of Radioengineering, Vol. 21, No. 1, 2012, pp. 312-316.

[20] S. Lawanwisut and M. Siripruchyanun, "An Active-Only High-Output Impedance Current-Mode Quadrature Oscillator Using CCCCTA Based-Lossless Sifferentiators," Proceedings of IEEE TENCON, Singapore, 23-26 January 2009, pp. 1-4.

[21] W. Jaikla and M. Siripruchyanun, "A Simple Oscillator Using only Single CCCCTA and Grounded Capacitors," Journal of Research and Development Rajamangala University of Technology, Tawan-ok, 2009, pp. 55-59.

[22] P. Silapan, T. Srisakul, W. Jaikla and M. Siripruchyanun, "Current Controlled CCTA (CCCCTA) Based-Novel Floating and Grounded Inductance Simulators," Journal of Research and development Rajamangala University of Technology, Tawan-ok, 2009, pp. 48-54.

[23] A. Toker, O. Cicekoglu, S. Ozacan and H. Kuntman, "High Output Impedance Transadmittance type Continuous Time Multifunction Filter with Minimum Active Elements," International Journal of Electronics, Vol. 88, No. 10, 2001, pp. 1085-1091 doi:10.1080/00207210110071260

[24] M. T. Abuelma'atti and A. Bentrcia, "Multi-Function Active-Only Current-Mode Filter with Three Input and One Output," International Journal of Electronics, Vol. 85, No. 4, 1998, pp. 431-435. doi:10.1080/002072198133978

[25] S. Lawanwisut and M. Siripruchyanun, "Active-Only Current-Mode KHN Filter with Electronic Controllability," The 3rd International Conference on Technical Education, 2010, pp. 46-51.

[26] D. R. Frey, "Log-Domain Filtering: An Approach to Current Mode Filtering," Proceedings of IEEE Circuits, Devices and Systems, Vol. 140, No. 6, 1993, pp. 406-416. doi:10.1049/ip-g-2.1993.0066 